\title{
On the Effectiveness of Suspension Stems in Reducing the Vibration Transmitted to a Cyclist's Hands in Road Cycling ${ }^{+}$
}

\author{
Jean-Marc Drouet ${ }^{1, *}$, Derek Covill ${ }^{2}$ and Antoine Labrie ${ }^{1}$ \\ 1 VÉLUS Laboratory, Mechanical Engineering Department, Université de Sherbrooke, \\ 2500 Boulevard de l'Université, Sherbrooke, QC J1K 2R1, Canada; antoine.labrie@usherbrooke.ca \\ 2 School of Computing, Engineering and Mathematics-University of Brighton, Cockcroft Building, \\ Lewes Road, Brighton BN2 4GJ, UK; d.covill@brighton.ac.uk \\ * Correspondence: jean-marc.drouet@usherbrooke.ca; Tel.: +1-819-821-8000 (ext. 61345) \\ + Presented at the 13th conference of the International Sports Engineering Association, Online, \\ 22-26 June 2020.
}

Published: 15 June 2020

\begin{abstract}
The practice of road cycling is often associated with low levels of comfort for the cyclist and can be a physically painful experience on bad roads. Apart from cushioning in the saddle, applying handlebar tape, or reducing tyre pressure, a road bicycle offers in itself few options for comfort improvement, as it is primarily designed for performance, with emphasis on low mass and high stiffness. However, a range of components exist (e.g., suspension stems and seatposts) that can be fitted to a road bicycle, which can potentially improve comfort. In this context, the aim of this study was to assess the effectiveness of suspension stems in reducing the vibration transmitted to a cyclist's hands in the case of impact loading. The results showed an important reduction in the vibrational energy transmitted to a cyclist's hands with two commercially available suspension stems compared to a regular stem.
\end{abstract}

Keywords: road bicycle; dynamic comfort; vibration; suspension stem; transmitted energy

\section{Introduction}

The practice of road cycling is often associated with low levels of comfort for the cyclist and can be a physically painful experience on bad roads. It can also be linked to increased health risks such as handlebar palsy caused by the compression of the ulnar nerve [1], and the potential onset of erectile dysfunction caused by compression of the pudendal nerve [2]. In that regard, reducing road induced vibration transmitted to the cyclist is an important consideration, and has been an increasingly active research field since the early 2000's. Many studies have been undertaken to assess and understand road bicycle dynamic comfort, which relates to the capacity of a road bicycle to filter the vibration generated by the road surface, and this must be distinguished from static comfort, which relates to the bicycle's size with respect to the size and shape of the cyclist. A range of test protocols and test rigs have been developed that mimic the excitation of a road surface [3-6], and transducers have commonly been used around the bicycle, e.g., in the seatpost, handlebar, stem, and brake hoods in order to measure the acceleration, force, power, and energy transmitted to the cyclist [7-10]. An assessment of the ability of bicycles, bicycle components, and cycling apparel to filter vibration has also been conducted [3,5,10-12], while cyclists' vibration perceptual thresholds have been determined using psychophysical approaches [13,14].

Apart from cushioning in the saddle, applying handlebar tape, or reducing tyre pressure, a road bicycle offers in itself few options for comfort improvement, as it is primarily designed for 
performance, with emphasis on low mass and high stiffness, and typically is not equipped with suspension components, as is the case for other types of bicycles (e.g., full suspension mountain bike). However, a range of components exist (e.g., suspension stems and seatposts) that can be fitted to a road bicycle, which can potentially improve comfort with only a limited increase in mass.

In this context, the aim of this study was to assess the effectiveness of suspension stems in reducing the vibration transmitted to a cyclist's hands in the case of impact load excitation at the front wheel. We chose to use impact loads as it was thought that these would provide a more measurable difference between test conditions than random-type excitation (i.e., excitation related to road surface roughness). The vibrational energy transmitted at a cyclist's hands was used for this assessment, as in previous studies for impact load excitation $[12,14]$.

\section{Materials and Methods}

Three $90 \mathrm{~mm}$ road bicycle stems were tested in this study (Figure 1), namely: (1) a Kalloy Uno 7 stem (Model ASA105, Kalloy Industrial, Taiwan), (2) a TranzX ANTISHOCK suspension stem (model JD-ST146A.2, JD Components Co., Ltd., Taiwan), and (3) a Redshift ShockStop suspension stem (model RS-40-01, RedShift Sports, Philadelphia, PA, USA). The technical data for the three stems are given in Table 1. The suspensions stems were selected for testing in this study because they were deemed the two most suitable suspension stems for road bike application. The Kalloy Uno 7 stem is a regular stem (i.e., not a suspension stem), and this was used as the reference stem in this study. Both suspension stems are made of two main articulated parts that rotate relatively to one another about a horizontal axis (i.e., $a-a$ axis in Figure $1 b, c)$. One of these parts is attached to the fork steerer tube and the other is attached to the handlebar. An elastomer component is inserted between them, providing compliance and damping to the system. The elastomer component for the Redshift ShockStop stem is interchangeable, and, in this study, was selected to match the cyclist's mass according to the manufacturer's guidelines. The elastomer component for the TranzX ANTISHOCK stem is not interchangeable.

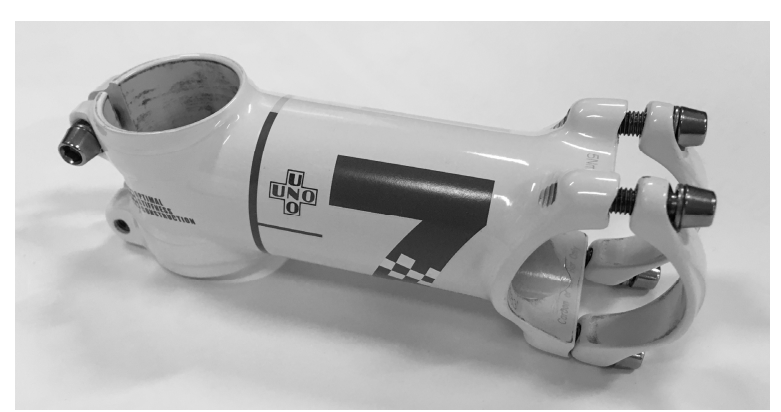

(a)

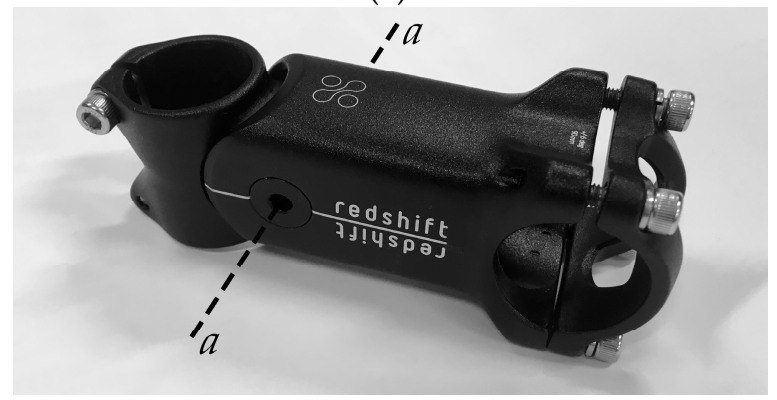

(c)

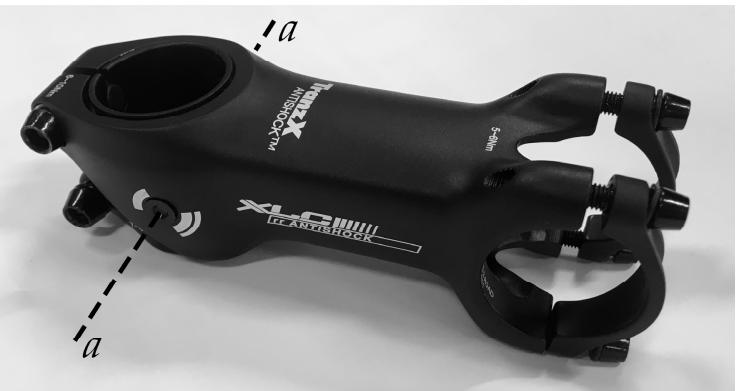

(b)

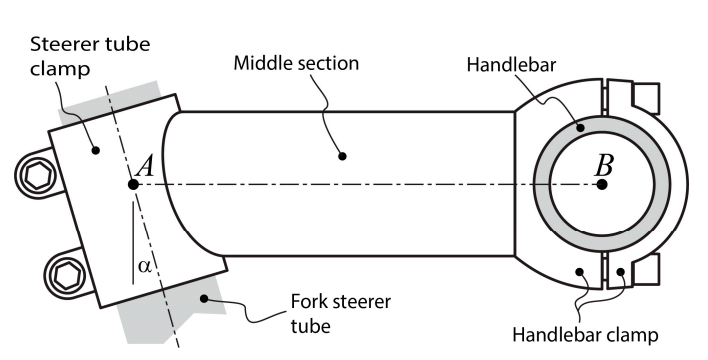

(d)

Figure 1. Tested stems: (a) Kalloy Uno 7, (b) TranzX ANTISHOCK, (c) Redshift ShockStop, and (d) diagram showing the relevant parts of the stem. 
Table 1. Technical data for the tested stems, with reference to Figure 1d.

\begin{tabular}{lccc}
\hline & Kalloy Uno 7 & $\begin{array}{c}\text { TranzX } \\
\text { ANTISHOCK }\end{array}$ & $\begin{array}{c}\text { Redshift } \\
\text { ShockStop }\end{array}$ \\
\hline Length (distance $A B)(\mathbf{m m})$ & 90 & 90 & 90 \\
Angle $\boldsymbol{\alpha}\left({ }^{\circ}\right)$ & 7 & 7 & 6 \\
Steerer tube diameter $(\mathbf{m m})$ & 28.6 & 28.6 & 28.6 \\
Handlebar diameter $(\mathbf{m m})$ & 31.8 & 31.8 & 31.8 \\
Distance between point $\boldsymbol{A}$ and & - & 4 & 26 \\
$\boldsymbol{a}-\boldsymbol{a}$ axis along $\boldsymbol{A B}$ line $(\mathbf{m m})$ & 103 & 192 & 271 \\
Mass (g) & 7050 & $2014-\mathrm{T} 6$ & $6061-\mathrm{T} 6$ \\
Material (aluminium alloy) & & & \\
\hline
\end{tabular}

Tests were carried out using a bicycle treadmill (Figure 2a) and a Cervélo R3 carbon fibre road bicycle (size: $56 \mathrm{~cm}$; tyre: Continental Grand Prix 4000s II-700×23C; pressure: $800 \mathrm{kPa}$ ). The treadmill platform was $76 \mathrm{~cm}$ wide by $196 \mathrm{~cm}$ long, and allowed for ample freedom of movement for cycling. The rear wheel was placed on a raiser board and was thus not touching the treadmill belt. Impact load excitation at the front wheel was provided using a circular aluminium dowel of $15.7 \mathrm{~mm}$ in diameter, attached to the treadmill belt. The bicycle and the cyclist were kept vertically stable with an elastic rope wrapped around the seat tube and attached to a fixed structure on each side of the treadmill. The elastic rope was selected to be compliant enough in the vertical direction in order to make sure it did not affect the bicycle's dynamics in that direction.

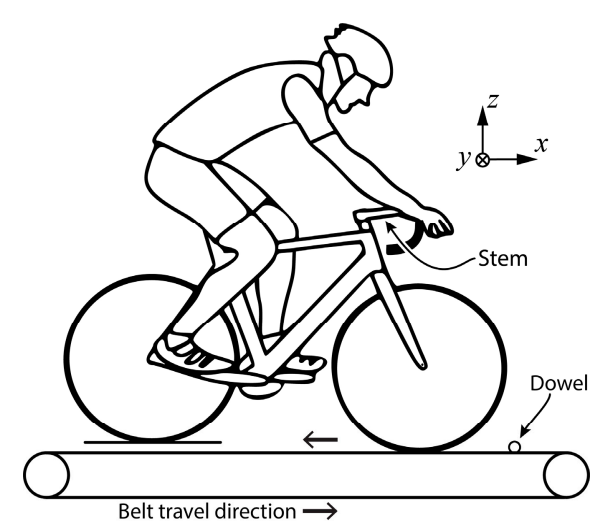

(a)

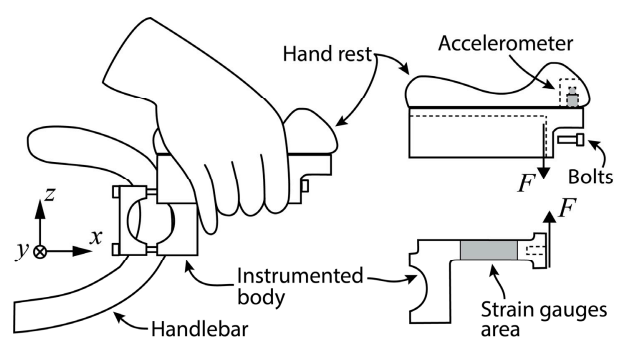

(b)

Figure 2. (a) Bicycle treadmill with a dowel attached to the belt. (b) Instrumented brake hood [10,15].

The vertical force and acceleration transmitted to the cyclist's hands were measured with a strain gauge instrumented brake hood [10] and a PCB 352C68 uniaxial accelerometer under the hands (Figure $2 b$ ). Each instrumented brake hood was composed of two main parts bolted together-the hand rest and the instrumented body equipped with strain gauges in a full Wheatstone bridge configuration. The instrumented brake hoods were statically and dynamically calibrated for transmitted power measurements at a cyclist's hands [15]. Because of their high stiffness, their deflection in the $x-z$ plane did not introduce any significant inclination of the measurement axis relative to the $z$ axis (i.e., $<0.05^{\circ}$ at maximum load), and therefore did not impair the force and acceleration measurements along that axis. The force and acceleration signals were collected using a LMS SCADAS 24-bit acquisition system (model SCR01-08B) at a sampling frequency of $8192 \mathrm{~Hz}$. LMS Test.Lab software was used for the data processing. The instantaneous power transmitted to the cyclist's hands, $P$, was calculated using Equation (1), where $F$ and $v$ are the instantaneous vertical force and speed, respectively (obtained by integrating the acceleration signal).

$$
P(t)=F(t) v(t)
$$

The energy transmitted to the cyclist's hands, E, for each impact was calculated using Equation (2). 


$$
E=\int P(t) d t
$$

An experienced male cyclist (age: 49 years old; height: $180 \mathrm{~cm}$; mass: $77 \mathrm{~kg}$ ) was seated on the bicycle during the measurements. The bicycle was properly adjusted to achieve an adequate body position. The cyclist kept a natural constant position on the bicycle with his hands on the instrumented brake hoods with no grip force. He remained seated at all times and did not pedal. The bicycle cranks were fixed in a horizontal position.

Three runs of 20 impacts per stem were carried out for a total 60 impacts per stem. The order of the runs was randomised across the stems. For each stem, the average total (i.e., left hand (LH) and right hand (RH) combined) transmitted energy, $\bar{E}$, was calculated using Equation (3).

$$
\bar{E}=\frac{1}{60} \sum_{i=1}^{60}\left(E_{\mathrm{LH}, i}+E_{\mathrm{RH}, i}\right)
$$

where $E_{\mathrm{LH}, i}$ and $E_{\mathrm{RH}, i}$ are the transmitted energy measured by the left and right instrumented brake hoods, respectively, for the $i^{\text {th }}$ impact.

For comparison purposes, using the same methodology, the average total transmitted energy was also assessed for the front tyre pressure, ranging from 300 to $800 \mathrm{kPa}$ with $100 \mathrm{kPa}$ increments. This assessment was carried out for the Kalloy Uno 7 stem only.

\section{Results}

The average total transmitted energy, $\bar{E}$, for each of the three stems is shown in Figure 3a. The average total transmitted energy as a function of the front tyre pressure for the Kalloy Uno 7 stem is shown in Figure $3 \mathrm{~b}$ (solid line). The average total transmitted energy for the three stems at a front tyre pressure of $800 \mathrm{kPa}$ is indicated by the dashed lines.

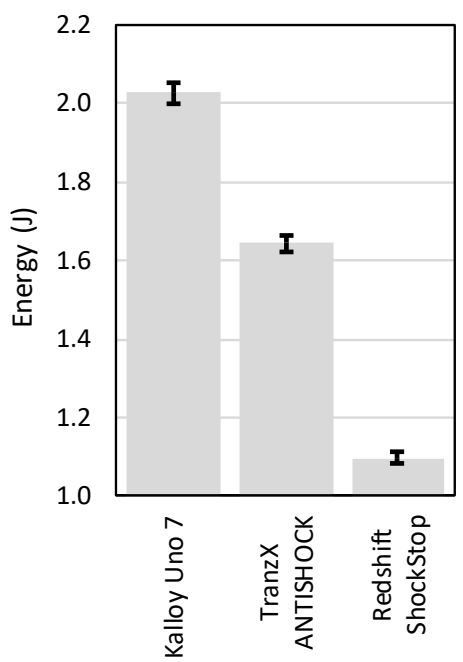

(a)

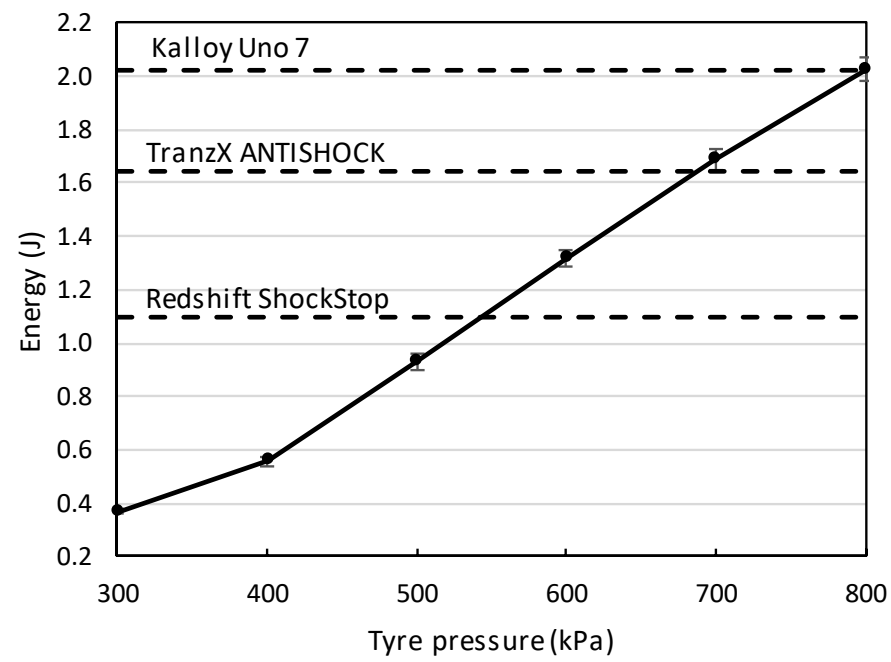

(b)

Figure 3. (a) Average total transmitted energy for the three stems. (b) Average total transmitted energy as a function of the front tyre pressure for the Kalloy Uno 7 stem (solid line). The average total transmitted energy for the three stems at a front tyre pressure of $800 \mathrm{kPa}$ is indicated by the dashed lines. Uncertainty bars are at a confidence level of $95 \%$.

Table 2 gives the change in $\bar{E}$ and $p$-values (t-test for mean values) for pair-wise comparisons between the three stems. 
Table 2. Change in average total transmitted energy to the cyclist's hands for pair-wise comparisons between the three stems.

\begin{tabular}{lccc}
\hline \multicolumn{1}{c}{ Stem Pairs } & $\Delta \bar{E}$ & $\Delta \bar{E}$ & $\begin{array}{c}p \text {-Value } \\
\text { for }\end{array}$ \\
\hline 1. Redshift ShockStop vs Kalloy Uno 7 & -0.928 & -45.8 & $<0.001$ \\
2. TranzX ANTISHOCK vs Kalloy Uno 7 & -0.383 & -18.9 & $<0.001$ \\
3. Redshift ShockStop vs TranzX ANTISHOCK & -0.545 & -33.2 & $<0.001$ \\
\hline
\end{tabular}

\section{Discussion}

The results show an important reduction in vibrational energy transmitted to the cyclist's hands when using the suspension stems compared with the regular stem (i.e., Kalloy Uno 7 stem). Indeed, as disclosed in Table 2, the transmitted energy was reduced by $18.9 \%$ when using the TranzX ANTISHOCK stem, and was almost halved (-45.8\%) when using the Redshift ShockStop stem. In the tested conditions, the Redshift ShockStop stem was therefore found to be more than twice as efficient in reducing transmitted energy than the TranzX ANTISHOCK stem. The reduction in transmitted energy obtained with the suspension stems is also much more important than the one obtained in a previous study in similar test conditions when using cycling gloves or handlebar tape. In that study, the reduction in transmitted energy was at most (i.e., when gloves and handlebar tape were used together) $8.3 \%$ [14].

Figure $3 \mathrm{~b}$ allows us to relate the reduction in transmitted energy to the cyclist's hands using the instrumented stems to a change in front tyre pressure, which will yield a similar reduction in transmitted energy. This comparison is meaningful to cyclists, as tyre pressure has a direct effect on their perception of bicycle comfort [11]. Tyre pressure is also a parameter that cyclists are likely to have made adjustments to in an attempt to improve comfort or performance, and reducing tyre pressure is the quickest way to reduce transmitted energy to the cyclist-thereby increasing comfort. However, there is a potential trade-off between tyre pressure and other considerations, such as the rolling resistance. Figure $3 \mathrm{~b}$ shows that in order to obtain a reduction in transmitted energy comparable to that obtained with the TranzX ANTISHOCK and Redshift ShockStop stems (compared to the regular stem), a deflation of the front tyre from $800 \mathrm{kPa}$ (or $116 \mathrm{psi}$ ) to $\sim 700 \mathrm{kPa}$ (or $\sim 102 \mathrm{psi}$ ) and $\sim 550 \mathrm{kPa}$ (or $\sim 78 \mathrm{psi}$ ), respectively, would be required. So, the suspension stems allow for a reduction of transmitted energy associated with deflating the front tyre somewhere between 100 and $250 \mathrm{kPa}$ (depending on the stem), without having to compromise on other considerations related to tyre pressure selection.

The measured reduction in transmitted energy associated with both suspension stems (i.e., 383 $\mathrm{mJ}$ and $928 \mathrm{~mJ}$ for the TranzX ANTISHOCK stem and the Redshift ShockStop stem, respectively) was vastly higher than the $100 \mathrm{~mJ}$ perceptual threshold determined in a previous study involving 10 participants and carried out in similar test conditions [12]. It is therefore expected that cyclists will perceive an improvement in comfort when using the tested suspension stems. However, the added comfort provided by these stems comes at the expense of the mass of this component, due to their increased mechanical complexity. The suspension stems were 1.9 and 2.6 times heavier (for the TranzX ANTISHOCK stem and the Redshift ShockStop stem, respectively) than the regular stem, which, in absolute terms, correspond to mass increases of $89 \mathrm{~g}$ and $168 \mathrm{~g}$, respectively, when compared to the regular stem (103 g). This should be an area for further study, focusing on how the increase in mass and other properties (e.g., stiffness and damping characteristics) of suspension stems affect the amount of energy transmitted to the cyclist.

\section{Conclusions}

It is clear that using a suspension stem is an effective means of reducing road-induced vibration transmitted to the hands in impact loading conditions; however, the performance of suspension stem products can vary considerably. Both of the suspension stem products tested in this study demonstrated a large reduction in the total transmitted energy, and this was vastly higher than the 
perceptual threshold of cyclists as determined in a previous study in similar test conditions. The cumulative effect of the many impacts on the body that occur during a typical ride could be considerable, which suggests that there is a strong incentive for cyclists to use a suspension stem. Future studies should focus on assessing the effectiveness of suspension stems in the case of randomtype excitation at the front wheel, and the effectiveness of suspension seatposts in reducing roadinduced vibration transmitted to the buttocks.

Conflicts of Interest: The authors declare no conflict of interest.

\section{References}

1. Akuthota, V.; Plastaras, C.; Lindberg, K.; Tobey, J.; Press, J.; Garvan, C. The effect of long-distance bicycling on ulnar and median nerves: An electrophysiologic evaluation of cyclist palsy. Am. J. Sports Med. 2005, 33, 1224-1230.

2. Sommer, F.; Goldstein, I.; Korda, J.B. Bicycle Riding and Erectile Dysfunction: A Review. J. Sex. Med. 2010, 7, 2346-2358.

3. Petrone, N.; Giubilato, F. Development of a Test Method for the Comparative Analysis of Bicycle Saddle Vibration Transmissibility. Procedia Eng. 2013, 60, 288-293.

4. Lépine, J.; Champoux, Y.; Drouet, J.-M. Road bicycle comfort: On the measurement of vibrations induced to cyclist. Sports Eng. 2014, 17, 113-122.

5. Petrone, N.; Trabacchin, F. Development of a test bench for the subjective \& objective evaluation of the vibrational comfort of bicycle components. In Proceedings of the ASME Design Engineering Technical Conferences, Buffalo, NY, USA, 17-20 August 2014.

6. Lépine, J.; Champoux, Y.; Drouet, J.-M. Test Protocol for In-situ Bicycle Wheel Dynamic Comfort Comparison. Procedia Eng. 2016, 147, 568-572.

7. Drouet, J.-M.; Champoux, Y. A novel dynamometric hubset design to measure wheel loads in road cycling. Procedia Eng. 2010, 2, 2925-2930.

8. Drouet, J.-M.; Champoux, Y. Development of a three-load component instrumented stem for road cycling. Procedia Eng. 2012, 34, 502-507.

9. Vanwalleghem, J.; De Baere, I.; Loccufier, M.; Van Paepegem, W. Sensor design for outdoor racing bicycle field testing for human vibration comfort evaluation. Meas. Sci. Technol. 2013, 24, 095002.

10. Lépine, J.; Champoux, Y.; Drouet, J.-M. The relative contribution of road bicycle components on vibration induced to the cyclist. Sports Eng. 2015, 18, 79-91.

11. Marcolin, G.; Petrone, N.; Reggiani, C.; Panizzolo, F.A.; Paoli, A. Biomechanical Comparison of Shorts with Different Pads: An Insight into the Perineum Protection Issue. Medicine 2015, 94, e1186.

12. Drouet, J.-M.; Covill, D.; Duarte, W. On the Exposure of Hands to Vibration in Road Cycling: An Assessment of the Effect of Gloves and Handlebar Tape. Proceedings 2018, 2, 213.

13. Richard, S.; Champoux, Y.; Lépine, J.; Drouet, J.-M. Using an alternative forced-choice method to study shock perception at cyclists' hands: The effect of tyre pressure. Procedia Eng. 2015, 112, 361-366.

14. Drouet, J.-M.; Guastavino, C.; Girard, N. Perceptual Thresholds for Shock-type Excitation of the Front Wheel of a Road Bicycle at the Cyclist's Hands. Procedia Eng. 2016, 147, 724-729.

15. Champoux, Y.; Vanwalleghem, J.; Drouet, J.-M. Dynamic calibration of an instrumented bike brake hood in measuring power absorbed by the hands. Procedia Eng. 2015, 112, 225-230.

(C) 2020 by the authors. Licensee MDPI, Basel, Switzerland. This article is an open access article distributed under the terms and conditions of the Creative Commons Attribution (CC BY) license (http://creativecommons.org/licenses/by/4.0/). 